\title{
Mediation of Movement-Induced Breakthrough Cancer Pain by IB4-Binding Nociceptors in Rats
}

\author{
Joshua Havelin, ${ }^{1}$ Ian Imbert, ${ }^{1}$ Devki Sukhtankar, ${ }^{2}$ Bethany Remeniuk, ${ }^{2}$-Ian Pelletier, ${ }^{1}$ Jonathan Gentry, ${ }^{1}$ Alec 0kun, ${ }^{3}$ \\ Timothy Tiutan, ${ }^{3}$ Frank Porreca, ${ }^{2,3}$ and Tamara E. King ${ }^{1}$ \\ ${ }^{1}$ Department of Biomedical Sciences, College of Osteopathic Medicine, Center for Excellence in the Neurosciences, University of New England, Biddeford, \\ Maine 04005, and ${ }^{2}$ Department of Cancer Biology, Arizona Cancer Center, and ${ }^{3}$ Department of Pharmacology, College of Medicine, University of Arizona, \\ Tucson, Arizona 85724
}

Cancer-induced bone pain is characterized by moderate to severe ongoing pain that commonly requires the use of opiates. Even when ongoing pain is well controlled, patients can suffer breakthrough pain (BTP), episodic severe pain that "breaks through" the medication. We developed a novel model of cancer-induced BTP using female rats with mammary adenocarcinoma cells sealed within the tibia. We demonstrated previously that rats with bone cancer learn to prefer a context paired with saphenous nerve block to elicit pain relief (i.e., conditioned place preference, CPP), revealing the presence of ongoing pain. Treatment with systemic morphine abolished CPP to saphenous nerve block, demonstrating control of ongoing pain. Here, we show that pairing BTP induced by experimenter-induced movement of the tumor-bearing hindlimb with a context produces conditioned place avoidance (CPA) in rats treated with morphine to control ongoing pain, consistent with clinical observation of BTP. Preventing movement-induced afferent input by saphenous nerve block before, but not after, hindlimb movement blocked movement-induced BTP. Ablation of isolectin B4 (IB4)-binding, but not TRPV1 ${ }^{+}$, sensory afferents eliminated movement-induced BTP, suggesting that input from IB4-binding fibers mediates BTP. Identification of potential molecular targets specific to this population of fibers may allow for the development of peripherally restricted analgesics that control BTP and improve quality of life in patients with skeletal metastases.

Key words: breakthrough pain; c-fiber; cancer pain; IB4; nonpeptidergic; peptidergic

\section{Significance Statement}

We present a novel preclinical measure of movement-induced breakthrough pain (BTP) that is observed in the presence of morphine controlling ongoing pain. Blockade of sensory input before movement prevented BTP, whereas nerve block after movement failed to reverse BTP. These observations indicate that blocking peripheral sensory input may prevent BTP and targeting central sites may be required for pain relief once BTP has been initiated. Preventing sensory input from TRPV1expressing fibers failed to alter movement-induced BTP. In contrast, preventing sensory input from isolectin B4 (IB4)-binding fibers blocked movement-induced BTP. Therefore, examining molecular targets on this population of nociceptive fibers may prove useful for developing an improved strategy for preventing BTP in cancer patients with skeletal metastases.

\section{Introduction}

Pain is the most feared consequence of cancer (Breivik et al., 2009; Paice and Ferrell, 2011). Metastatic bone pain is character-

\footnotetext{
Received April 10, 2016; revised March 30, 2017; accepted April 14, 2017.

Author contributions: F.P. and T.E.K. designed research; J.H., I.I., D.S., B.R., I.P., J.G., A.O., T.T., and T.E.K. performed research; J.H., I.I., D.S., B.R., F.P., and T.E.K. analyzed data; F.P. and T.E.K. wrote the paper.

This work was supported by the National Institutes of Health (National Cancer Institute Grant T32CA009213 to B.R., National Institutes on Drug Abuse Grant DA034975 to F.P., and National Institute of General Medical Sciences Grant P20GM103643 (PI: lan Meng; Project PI: T.E.K.).

The authors declare no competing financial interests.

Correspondence should be addressed to Tamara King, Ph.D., Department of Biomedical Sciences, College of Osteopathic Medicine, Center for Excellence in the Neurosciences, University of New England, Biddeford, ME 04043. E-mail: tking6@une.edu.
}

ized by moderate to severe persistent ongoing pain associated with tumor growth, nerve destruction, and bone remodeling. As many as $40-80 \%$ of these patients also experience breakthrough pain (BTP), transient episodes of severe to excruciating pain occurring in the presence of medication controlling background cancer pain (Portenoy and Hagen, 1989, 1990; Mercadante, 2015). BTP is frequently reported for $15-30$ min after voluntary or involuntary movements such as changing position or coughing, with as many as four to six episodes reported within a day dramatically reducing patients' quality of life (Haugen et al., 
2010; Mercadante, 2015). Treatment of BTP typically requires rapid-onset opioids, which although often effective, are confounded by uncertainty of dosing requirements for safe and effective treatment of individual patients (Mercadante, 2011, 2015). Further complicating treatment, BTP takes place on a background of medication, primarily opioid, controlled ongoing pain. Increasing the opioid dose results in a high likelihood of adverse side effects, further diminishing these patients' quality of life (Mercadante, 2015). The discovery of safe and effective medications to treat BTP is an urgent unmet medical need.

The observation that BTP occurs in the setting of opioid medication that sufficiently controls ongoing pain suggests that these pain states are mechanistically distinct (Bennett, 2010). Analgesic actions of drugs such as morphine occur at mu opioid receptors (MORs) that are located within the periphery, spinal cord, and brain. One possibility is that movement may recruit additional fibers that are not blocked by peripheral MOR agonists at doses that block persistent background pain. In the setting of paininduced central sensitization, such signals may elicit excruciating BTP (Mantyh, 2013; Falk and Dickenson, 2014; Mantyh, 2014) that requires fast-acting opioids that likely exert their effects at supraspinal sites.

Although recent RNA-sequencing (RNAseq) studies have described as many as 11 subpopulations of sensory fibers (Usoskin et al., 2015), two broad classes of fibers that have been widely characterized are TRPV1-expressing fibers and isolectin B4 (IB4)-binding neurons (Molliver et al., 1997; Snider and McMahon, 1998; Basbaum et al., 2009; Wang and Zylka, 2009). Previous studies have demonstrated that long-lasting desensitization of TRPV1-expressing sensory fibers produces insensitivity to thermal stimulation and ongoing pain without altering tactile hypersensitivity in models of neuropathic and inflammationinduced pain (Yaksh et al., 1979; Ossipov et al., 1999; Okun et al., 2011). Others have demonstrated that ablation of MAS related GPR family member D (MrgD)-expressing or IB4-binding fibers blocks tactile hypersensitivity without altering thermal responses (Joseph et al., 2008; Cavanaugh et al., 2009; Ye et al., 2012). Given the mechanical component of movement-evoked BTP, we tested the hypothesis that blocking nociceptive input from IB4-binding fibers will block movement-evoked BTP whereas blocking nociceptive input from TRPV1-expressing fibers will not block movement-evoked BTP.

Evaluation of BTP and cancer-induced ongoing pain has been limited by the lack of available preclinical models. Recently, we reported capturing ongoing cancer pain through a learning paradigm assessing motivation to seek a context associated with pain relief (Remeniuk et al., 2015). Here, we developed a novel measure of BTP by assessing the motivation to avoid a context associated (conditioned place avoidance, CPA) with pain after movement of the tumor-bearing hindlimb. Critically, this model assessed CPA in rats with morphine-controlled ongoing bone cancer pain, simulating movement-triggered BTP in patients.

\section{Materials and Methods}

\section{Animals}

Female and male Fischer F344/NhSD rats (Harlan Laboratories) weighing 125-150 g were chosen based on their histocompatibility with the MAT BIII tumor line. The rats were maintained on a $12 \mathrm{~h}$ light/dark cycle with food and water available ad libitum. All experiments were performed in accordance with the policies and recommendations of the International Association for the Study of Pain, the National Institutes of Health, and the Institutional Animal Care and Use Committee of the University of Arizona and University of New England.

\section{Cell line}

The Fischer rat mammary adenocarcinoma cell line 13762 MAT BIII (ATCC, CRL 1666, RRID:CVCL_3475) was maintained in McCoy's media with L-glutamine (CellGro) with $10 \%$ fetal bovine serum (ATCC) and $1 \%$ penicillin/streptomycin at $37^{\circ} \mathrm{C}$ in a $5 \% \mathrm{CO}_{2}$ atmosphere. Before surgical implantation, cells were washed with PBS and detached with $0.05 \%$ trypsin-EDTA (CellGro). The cells were centrifuged at $2300 \mathrm{rpm}$ for $3 \mathrm{~min}$ and resuspended at a concentration of $2 \times 10^{5} \mathrm{cells} / \mu \mathrm{l}$ in McCoy's serum-free medium.

\section{Surgical procedures and drug treatment}

Intratibial surgery and cancer implantation. This surgical procedure was performed according to previously published methods (Remeniuk et al., 2015). Briefly, rats were anesthetized under gas anesthesia ( $2 \%$ isoflurane- $\mathrm{O}_{2}$ mixture). The right hindlimb of the rat was shaved and disinfected with $70 \%$ ethanol and betadine. The animal was placed on its back, a $1 \mathrm{~cm}$ incision was made horizontally across the femoral-tibial region to expose the patellar tendon, and the surrounding skin was retracted to expose the proximal end of the tibia. A small hole was drilled between the lateral and medial condyles into the intramedullary canal and a $5 \mathrm{~cm}, 28$-gauge guide cannula was inserted (Plastics One) attached via Tygon tubing (Cole-Palmer) to a $25 \mu \mathrm{l}$ syringe (Hamilton) with location verified by radiographic imaging (Faxitron). Injection of $5 \mu \mathrm{l}$ of MAT BIII cells or cell-free McCoy's serum-free medium (vehicle), was followed by sealing the drilled hole with bone cement (Stryker Orthopaedics Simplex P Bone Cement). The area was flushed with sterile saline and the knee joint was reinforced with a vicryl 5-0 suture (Ethicon) placed across the drilled area. Each rat received $1 \mathrm{mg} / \mathrm{ml}$ gentamicin sulfate (Sparhawk Laboratories) via subcutaneous injection and was allowed to recover from anesthesia before being returned to the housing colony. Animals did not receive treatment with analgesics after tumor implantation because treatment with NSAIDs, specifically COX inhibitors, as well as morphine, have been demonstrated to affect aspects of disease progression, including tumor growth and tumor-induced bone remodeling (Sabino et al., 2002a; Sabino et al., 2002b; King et al., 2007). For ethical considerations, all experiments were terminated within $14 \mathrm{~d}$ of tumor inoculation into the tibia. A total of 83 tumor-bearing rats and 50 sham rats were used across all studies.

Morphine pellet implantation. Effects of morphine on tumor-induced bone pain were assessed by insertion of morphine or placebo pellets $11 \mathrm{~d}$ after intratibial surgery and behavioral testing 20-24 h after pellet implantation $12 \mathrm{~d}$ after intratibial surgery. Morphine sulfate $(75 \mathrm{mg})$ or placebo pellets, generously provided by the National Institute on Drug Abuse (NIDA) Drug Supply Program, were inserted subcutaneously under gas anesthesia ( $2 \%$ isoflurane $\mathrm{O}_{2}$ mixture) on the side contralateral to tibia surgery of the rat in front of the pelvic region. The region was shaved and disinfected with $70 \%$ ethanol and betadine. A $1 \mathrm{~cm}$ incision was made into the skin and a pocket was created with forceps in between the skin and muscle. A single pellet was inserted into the pocket region and the incision was closed using a surgical wound clip. Rats received $1 \mathrm{mg} / \mathrm{ml}$ of subcutaneous gentamicin sulfate (Sparhawk Laboratories) and were allowed to recover from anesthesia before being returned to the housing colony. For conditioned place preference (CPP) and CPA experiments, pellets were implanted immediately after assessment of baseline (preconditioning) time spent in the conditioning chambers.

Intrathecal catheterization and spinal drug delivery. Rats underwent surgical implantation of an intrathecal catheter for drug administration at the level of the lumbar spinal cord a minimum of $7 \mathrm{~d}$ before intratibial surgeries to allow sufficient recovery time from the intrathecal surgeries. Intrathecal catheters were implanted surgically as described previously (Yaksh and Rudy, 1976; Yaksh et al., 1979; King et al., 2011). Rats were anesthetized under gas anesthesia ( $2 \%$ isoflurane $\mathrm{O}_{2}$ mixture) while secured in a stereotaxic frame (Stoelting). The atlanto-occipital membrane was exposed, punctured, and a section of polyethylene-10 tubing $6.5 \mathrm{~cm}$ in length was passed caudally from the cisterna magna to the lumbar enlargement for a single spinal administration of the appropriate drug or vehicle. To determine the effect of eliminating input from TRPV1-expressing fibers, rats received spinal administration of capsaicin (20 $\mu \mathrm{g} / 10 \mu \mathrm{l} 10 \%$ Tween, $10 \%$ ethanol, $80 \%$ saline) followed by $10 \mu \mathrm{l}$ 
saline flush or equivolume vehicle (10\% Tween, $10 \%$ ethanol, $80 \%$ saline) followed by $10 \mu \mathrm{l}$ saline flush. To determine the effect of eliminating input from IB4-binding fibers, separate groups of rats received spinal administration of IB4-Saporin (IB4-SAP, $3.2 \mu \mathrm{g} / 20 \mu \mathrm{l}$ saline; Advanced Targeting Systems) or the control, blank-SAP $(3.2 \mu \mathrm{g} / 20 \mu \mathrm{l}$ saline; Advanced Targeting Systems) followed by a $10 \mu \mathrm{l}$ flush of saline. Movement of an air bubble placed between drug solution and saline was used to monitor progress of the injection. Immediately after injection, catheters were slowly removed from the spinal cord and the wound was closed. Any animals displaying motor impairment or paralysis during recovery $(<10 \%$ total rats) were immediately euthanized. Animals were routinely checked throughout the experiment to monitor health. After behavioral testing, rats were euthanized and tissue collected for immunohistochemical verification of elimination of TRPV1 or IB4 immunofluorescence in the spinal dorsal horn of capsaicin or IB4-SAP-treated rats, respectively.

Immunofluorescent analysis of effect of spinal capsaicin or IB4-SAP on TRPV1, SP, CGRP, and IB4 immunofluorescence in the spinal dorsal horn. To verify that these treatments produced selective ablations of targeted nociceptor terminals, tissue was collected for immunofluorescent staining and semiquantitative image analysis $21 \mathrm{~d}$ after intrathecal administration of IB4-saporin, blank-SAP, capsaicin, or the vehicle for capsaicin. Rats were deeply anesthetized with Beuthanasia-D (Henry Schein Animal Health) and underwent intracardiac perfusion through the left ventricle with PBS containing heparin $(100 \mathrm{U} / \mathrm{ml})$ followed by $4 \%$ PFA containing PBS, pH 7.4. The L3-L4 spinal cord segments were immediately dissected out and postfixed in $10 \%$ formalin overnight. The spinal cord was then moved into a $30 \%$ sucrose solution at $4^{\circ} \mathrm{C}$ for $12-24 \mathrm{~h}$ for cryoprotection. Spinal cords were embedded in optimal cutting temperature (OCT) medium (VWR) and frozen on dry ice for sectioning. Sections were cut on a cryostat (Leica) at $30 \mu \mathrm{m}$, collected onto positively charged slides (Azer Scientific), and allowed to dry before storage at $-80^{\circ} \mathrm{C}$. Sections were rinsed 3 times with PBS containing $0.05 \%$ Tween (PBST) to remove OCT medium, and then nonspecific binding proteins were blocked by $30 \mathrm{~min}$ of incubation with $5 \%$ normal donkey serum (EMD Millipore) and 1\% bovine serum albumin (Amresco) in PBST. This blocking solution was also the antibody diluent. Primary antibodies and Alexa Fluor 488-conjugated IB4 were incubated overnight at $4^{\circ} \mathrm{C}$ as follows: IB4-AF488 1:500 (Invitrogen catalog \#I21411 also I21411 RRID: AB_2314662); goat anti-TRPV1 1:100 (R\&D Systems catalog \#AF3066); rabbit anti-Substance P 1:500 (ImmunoStar catalog \#20064, RRID: AB_572266); rabbit anti-CGRP 1:2000 (ImmunoStar catalog \#24112, RRID:AB_572217). The TRPV1 antibody was validated in rat DRG tissue by competition experiments with the TRPV1 antigen peptide that completely abolished binding of the TRPV1 antibody and it was verified that the antibody detected a protein of the appropriate molecular weight in Western blots (Isensee et al., 2014). Both the CGRP and SP antibodies are widely used and cited in the literature, with 504 citations for the SP antibody and 110 citations for the CGRP antibody, verifying that these antibodies are widely used in peer-reviewed journals (https://www. citeab.com/), proposed as the most reliable way to identify a suitable antibody (Helsby et al., 2014). Sections were rinsed 3 times with PBST and incubated in the dark for $1 \mathrm{~h}$ at room temperature with the appropriate cross-adsorbed secondary antibodies: donkey anti-goat Alexa Fluor 568 1:1000 (A-11057; Life Technologies); donkey anti-rabbit Alexa Fluor 568 1:1000 (ab175692; Abcam); and donkey anti-goat Alexa Fluor 488 1:1000 (ab150133; Abcam). Sections were rinsed with PBS three times and mounted with DAPI-containing Fluoroshield (ab104139; Abcam). Primary and/or secondary antibody omission controls under identical staining conditions resulted in no fluorescent signal.

Images were acquired using a Leica TCS SP5 confocal laser scanning microscope using a 20×/0.7 numerical aperture plan Apo objective lens. Excitation light was generated at $488 \mathrm{~nm}$ by an argon gas laser or $561 \mathrm{~nm}$ by a diode-pumped solid-state laser through a double dichroic beam splitter and emission was detected sequentially via photomultiplier tubes to avoid cross talk between fluorophores. $z$-stack images were collected with a $0.71 \mu \mathrm{m}$ step size and maximum projections generated for subsequent analysis. All $z$-stack images within each staining condition were acquired in a single session using the same laser intensity settings. Images were analyzed using FIJI image analysis software (Schindelin et al., 2012).
Table 1. Numbers of sections analyzed for staining intensity

\begin{tabular}{lllll}
\hline & Sample 1 & Sample 2 & Sample 3 & Sample 4 \\
\hline TRPV1 & & & & \\
$\quad$ Control & 8 & 14 & 15 & \\
IB4-SAP & 6 & 9 & & \\
Capsaicin & 11 & 12 & 17 & \\
SP & & & & \\
Control & 13 & 15 & 15 & 18 \\
IB4-SAP & 9 & 9 & & \\
Capsaicin & 9 & 12 & 17 & \\
CGRP & & & & \\
Control & 12 & 13 & 16 & \\
IB4-SAP & 10 & 13 & & \\
Capsaicin & 7 & 13 & 17 & \\
IB4 & & & & \\
Control & 35 & 41 & & \\
IB4-SAP & 15 & 31 & 40 & \\
Capsaicin & 27 & 36 & & \\
\hline
\end{tabular}

Confocal $z$-stack images captured using the Leica confocal software were opened using the Bioformats plug-in. Images were rotated on the $x-/ y$ axis so that the dorsal portion of the spinal dorsal horn is located at the top of the image and a $z$-projection image was created using maximum intensity for 2D visualization of the stack. The split channels function was used to create red (TRPV1, SP, or CGRP) or green (IB4) fluorescent images. Regions of interest (ROIs) were selected from a file and arranged to calculate ratios of the mean gray value of pixels within the ROI for an area of lamina 1 and the same area in the deep dorsal horn (L1/L1con) and an area of lamina 2 and the same area in the deep dorsal horn (L2/L2con) (see Fig. 4). This allowed for an internal reference to normalize intensity ratings for comparison across sections. The number of sections analyzed for TRPV1 analyses are reported in Table 1. These numbers include sections from TRPV1/IB4, SP/IB4, and CGRP/ IB4 costains because there were no significant differences in intensity ratings for IB4 fluorescence across these three immunofluorescent conditions. Average intensity ratios across sections were used to calculate means and SEM across samples from individual animals. Ratios were calculated across spinal dorsal horn images from three control rats, four capsaicin-treated rats for SP and CGRP, three for TRPV1, and two IB4SAP-treated rats.

\section{Behavioral measures}

Behavioral measure of tactile hypersensitivity. Rats were placed into elevated chambers with a wire mesh floor and allowed to acclimate for 30 min. Paw withdrawal thresholds were determined in response to probing with calibrated von Frey filaments with spaced increments ranging from 0.5 to $15 \mathrm{~g}$. Each filament was applied to the middle of the plantar surface of the paw using the "up and down" method and analyzed using a Dixon nonparametric test (Chaplan et al., 1994).

Behavioral measure of impaired limb use. Limb use was assessed as described previously (Luger et al., 2001). The animal was placed in an empty pan and observed while walking. Usage of the treated limb was rated on the following scale: $0=$ complete lack of use, $1=$ partial nonuse, $2=$ limping and guarding, $3=$ limping, and $4=$ normal walking.

Behavioral measure of BTP using CPA. Analysis of movement-induced BTP was performed using CPA to a chamber associated with movement triggered BTP. Within the clinical setting, voluntary or involuntary movement has been reported to trigger episodes of BTP lasting $\sim 30 \mathrm{~min}$ after the movement (Haugen et al., 2010; Mercadante, 2015). Therefore, we hypothesized that placing the rat into the chamber after a $2 \mathrm{~min}$ period of movement of the tumor-bearing hindlimb would produce a transient period of BTP and that association of the BTP with the novel context produces motivation to avoid the chamber on test day resulting in CPA. CPA was assessed in a three-chamber apparatus with chambers distinguishable by visual, tactile, and odor cues. One pairing chamber within the apparatus had striped walls, a smooth floor, and pinklemonade lip balm (Lip Smacker; Markwins Beauty Products) applied to the ceiling. The other pairing chamber within the apparatus had uni- 
Table 2. Statistical analysis results for ANOVA

\begin{tabular}{|c|c|c|}
\hline Factor & $F_{(\mathrm{DF} 1, \mathrm{DF} 2)}$ & $p$-value \\
\hline \multicolumn{3}{|c|}{ Two-way ANOVA of tumor-induced tactile hypersensitivity (Figure $1 A$ ) } \\
\hline Interaction & $F_{(8,76)}=2.733$ & $p=0.0015$ \\
\hline Time & $F_{(4,76)}=11.23$ & $p<0.0001$ \\
\hline Sex & $F_{(2,19)}=5.46$ & $p<0.0134$ \\
\hline \multicolumn{3}{|c|}{ Two-way ANOVA of tumor-induced hindlimb impairment (Figure 1B) } \\
\hline Interaction & $F_{(8,76)}=7.238$ & $p<0.0001$ \\
\hline Time & $F_{(4,76)}=30.23$ & $p<0.0001$ \\
\hline Sex & $F_{(2,19)}=28.21$ & $p<0.0001$ \\
\hline \multicolumn{3}{|c|}{ Two-way ANOVA of BTP in morphine-pellet-treated rats (Figure 2B) } \\
\hline Interaction & $F_{(1,23)}=0.3347$ & $p=0.5685$ \\
\hline Tumor & $F_{(1,23)}=31.59$ & $p<0.0001$ \\
\hline Drug & $F_{(1,23)}^{(1,23)}=0.0409$ & $p=0.8415$ \\
\hline \multicolumn{3}{|c|}{ Two-way ANOVA of lidocaine pretreatment on BTP (Figure $3 A$ ) } \\
\hline Interaction & $F_{(1,24)}=20.26$ & $p<0.0001$ \\
\hline Tumor & $F_{(1,24)}^{(1,24)}=2.99$ & $p=0.0966$ \\
\hline Lidocaine & $F_{(1,24)}=26.01$ & $p<0.0001$ \\
\hline \multicolumn{3}{|c|}{ Two-way ANOVA of lidocaine posttreatment on BTP (Figure 3B) } \\
\hline Interaction & $F_{(1,18)}=0.1254$ & $p=0.7273$ \\
\hline Tumor & $F_{(1,18)}=19.42$ & $p=0.0003$ \\
\hline Lidocaine & $F_{(1,18)}=0.05562$ & $p=0.8162$ \\
\hline \multicolumn{3}{|c|}{ One-way ANOVA of IB4 intensity ratio (Figure 4M) } \\
\hline Treatment & $F_{(2,5)}=6.93$ & $p<0.036$ \\
\hline \multicolumn{3}{|c|}{ One-way ANOVA of TRPV1 intensity ratio (Figure $4 N$ ) } \\
\hline Treatment & $F_{(2,6)}=20.97$ & $p<0.002$ \\
\hline \multicolumn{3}{|c|}{ One-way ANOVA of SP intensity ratio (Figure 40) } \\
\hline Treatment & $F_{(2,6)}=8.82$ & $p<0.016$ \\
\hline \multicolumn{3}{|c|}{ One-way ANOVA of CGRP intensity ratio (Figure 4P) } \\
\hline Treatment & $F_{(2,6)}=5.85$ & $p<0.039$ \\
\hline \multicolumn{3}{|c|}{ Two-way ANOVA of Ablation effects on tactile hypersensitivity (Figure $5 A$ ) } \\
\hline Interaction & $F_{(2,47)}=2.518$ & $p<0.0914$ \\
\hline IB4 vs TRPV1 ablation & $F_{(2,47)}^{(2,4 /)}=29.68$ & $p<0.0001$ \\
\hline Tumor & $F_{(1,47)}^{(2,4 /)}=0.2817$ & $p<0.5981$ \\
\hline \multicolumn{3}{|c|}{ Two-way ANOVA of ablation effects on BTP (Figure 5B) } \\
\hline Interaction & $F_{(2,54)}=1.12$ & $p=0.3338$ \\
\hline Tumor & $F_{(2,54)}=5.199$ & $p=0.0086$ \\
\hline IB4 vs TRPV1 ablation & $F_{(1,54)}^{(2,34)}=1.065$ & $p=0.30368$ \\
\hline \multicolumn{3}{|c|}{ Two-way ANOVA of ablation effects on bone remodeling (Figure $6 B$ ) } \\
\hline Interaction & $F_{(1,21)}=0.6508$ & $p=0.4289$ \\
\hline Drug & $F_{(1,21)}^{(1,21)}=0.3508$ & $p=0.2715$ \\
\hline IB4 vs TRPV1 ablation & $F_{(1,21)}^{(1,21)}=0.0005$ & $p=0.9818$ \\
\hline
\end{tabular}

formly gray walls, a rough floor, and vanilla lip balm (Lip Smacker) applied to the ceiling. The neutral chamber had white walls, a smooth floor, no lip balm, and an LED light (Sylvania LED/DOTS/BLACK/1X12/ $\mathrm{BL})$ on the ceiling to diminish time spent in the middle chamber and encourage exploration of the pairing chambers. The boxes were cleaned with detergent (Sparkleen; Fisherbrand) between each baseline, conditioning, and testing session. Each rat underwent baseline, conditioning, and testing in the same CPP apparatus.

Both tumor-bearing and sham control rats underwent a $1 \mathrm{~d}$ preconditioning period (at d 11 after surgery) when they were placed in the 3 -chamber apparatus with open access to all chambers for $15 \mathrm{~min}$. Behavior was video recorded and time spent in each chamber determined by video-tracking software (Anymaze; Stoelting). Any rats spending $<180 \mathrm{~s}$ in a single chamber were removed from the study $(<25 \%$ total animals tested). On conditioning day (d 12), rats were paired with an enclosed chamber (striped or gray) for $30 \mathrm{~min}$ with no treatment to minimize potential accidental movement of the tumor-bearing hindlimb. Four hours later, rats underwent 2 min of movement to the cancerbearing limb and were placed in the opposite chamber. This treatment was demonstrated previously to induce spinal FOS and NK-1 receptor internalization in tumor-bearing mice, but not in sham-treated mice (Schwei et al., 1999). Hindlimb movements were completed in a separate room from the conditioning chambers to prevent other rats from being unnecessarily exposed to signs of distress from the rats during the hindlimb movement. Both sham and tumor-bearing rats vocalized during the treatment. On the test day (d 13), rats were once again placed in the three-chamber apparatus with open access to all chambers. Behavior was video recorded and time spent in each of the pairing chambers was determined with Anymaze video-tracking software. Difference scores were calculated as the preconditioning baseline $(\mathrm{BL})$ scores subtracted from test scores (test $-\mathrm{BL}$ ). A negative score indicates aversion and a positive score indicates preference.

Morphine's effects on BTP. To determine whether movement-induced CPA breaks through ongoing morphine treatment that controls ongoing pain (Remeniuk et al., 2015), the ability of morphine infusion across 20-24 h to block movement-induced CPA was determined. On day 11 after injection of cancer cells into the tibia, rats underwent baseline analysis of time spent in each of the conditioning chambers as described above, followed by implantation of extended release morphine or placebo pellets. Pellets were surgically implanted subcutaneously on the lower back 1 inch above the pelvic bone under isoflurane anesthesia immediately after preconditioning baselines were performed. The following day (conditioning day), movement of the tumor-bearing hindlimb was performed 20-24 h into morphine infusion.

Measure of sensory input on movement-induced BTP. To determine whether blocking sensory input blocks movement-induced BTP, rats underwent the $3 \mathrm{~d}$ CPA conditioning protocol as described above. On the morning of conditioning day, rats received saphenous saline $(350 \mu \mathrm{l})$ and 
no hindpaw movement of the tumor-bearing hindlimb and were then confined to the appropriate conditioning chamber. Afternoon conditioning occurred $4 \mathrm{~h}$ later. To determine whether saphenous lidocaine blocks movement-induced BTP, rats received saphenous lidocaine ( $4 \%$ $\mathrm{w} / \mathrm{v}, 350 \mu \mathrm{l}) 10 \mathrm{~min}$ before the $2 \mathrm{~min}$ movement of the tumor-bearing hindlimb and confinement within the opposite conditioning chamber. To determine whether blocking sensory input after hindlimb movement blocks movement-induced BTP, afternoon conditioning consisted of hindlimb movement for $2 \mathrm{~min}$, followed $10 \mathrm{~min}$ later by saphenous lidocaine and confinement to the opposite conditioning chamber. All saphenous injections were performed as described previously (Remeniuk et al., 2015). Rats were anesthetized with a $2 \%$ isoflurane- $\mathrm{O}_{2}$ mixture. To produce an effective peripheral nerve block, lidocaine was administered over the saphenous nerve in a single subcutaneous injection ( $4 \% \mathrm{w} / \mathrm{v}, 350$ $\mu \mathrm{l})$. Equivolume saline was given as a vehicle control.

Radiograph analysis of disease progression

Bones were rated according to a 4-point scale where $0=$ normal, nontumor-bearing bone with no bone remodeling; 1 = slight signs of bone remodeling; 2 = diffuse bone loss or pitting without full cortical bone loss; and 3 = clear pitting and full cortical bone loss.

\section{Statistical analysis}

All statistical analysis was performed and all graphs were made using GraphPad Prism 6.0 software. Group differences in tactile sensory thresholds were analyzed across time with a two-way ANOVA. Post hoc analysis of time-dependent changes after treatment was performed using the Bonferroni's multiple-comparisons test wherein each time point was compared with pretreatment values within each group. A probability level of 0.05 was used to establish significance. For CPA, the effects of treatment (cancer vs control) and conditioning chamber were analyzed by a two-way ANOVA. Sidak's multiple-comparisons tests were used for post hoc analysis of preconditioning (BL) versus postconditioning values within each treatment group. Group differences were analyzed by ANOVA using the difference scores that were calculated as postconditioning (test) preconditioning $(\mathrm{BL})$ time spent in the drug-paired chamber. A negative value indicates CPA and a positive value indicates CPP. Post hoc analysis was performed using Dunnett's multiple-comparisons test. Where appropriate, analysis was performed to determine whether the difference score was significantly different from zero using a one-sample $t$ test. Group differences in intensity ratios for immunofluorescence were determined using one-way ANOVAs followed by Dunnett's multiplecomparisons test. $F$ values and degrees of freedom for all ANOVAs are presented in Table 2.

\section{Results}

Tumor-induced bone loss, tactile hypersensitivity, and impaired limb use

Intratibial injection of rat breast cancer cells produced bone remodeling with bone loss apparent by d 12 after surgery in both male and female rats (Fig. 1A). Corresponding tactile hypersensitivity and impaired limb use was observed in both male and female rats (Fig. $1 B, C$, respectively). No overt differences in pain behaviors were observed between male and female rats. All subsequent experiments were performed in female Fischer 344/ NhSD rats.

\section{Movement-induced pain induces CPA that breaks through} morphine infusion

To determine whether movement of the tumor-bearing hindlimb induces a transient increase in pain intensity consistent with reports of BTP, rats underwent a single-trial conditioning protocol as outlined by the flowchart in Figure $2 A$. Movement of the tumor-bearing hindlimb significantly reduced time spent in the movement-paired chamber, indicating the presence of CPA selectively in the tumor-bearing rats (Fig. $2 A,{ }^{* *} p<0.01$ vs sham). Sham-treated control rats did not show any difference in time

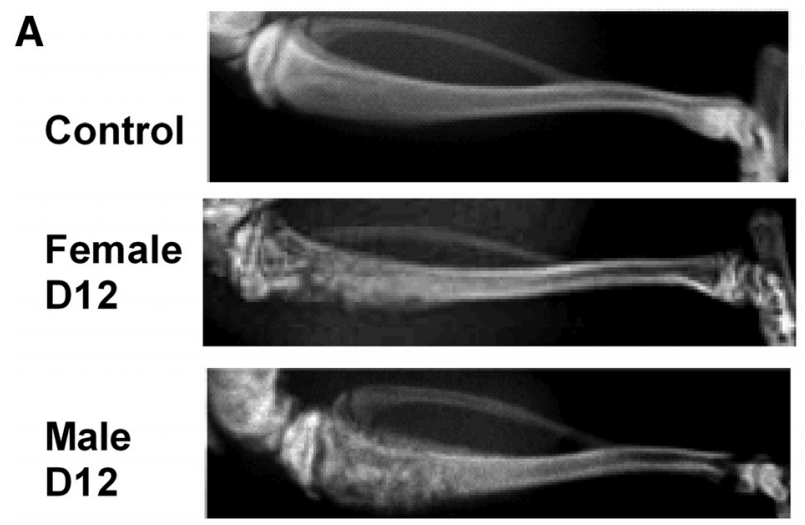

\section{Tactile Hypersensitivity}

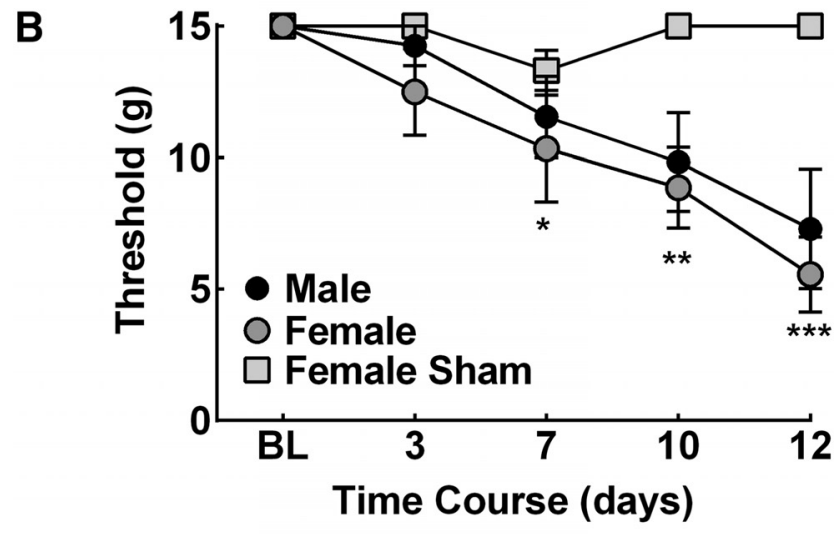

C

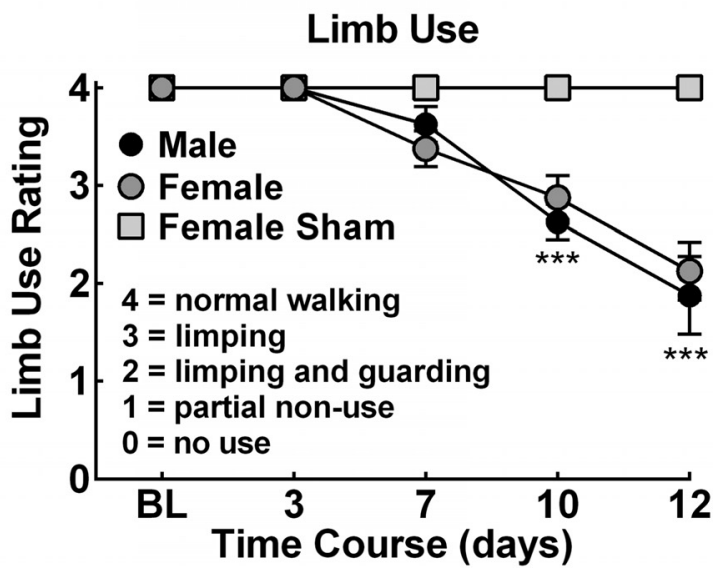

Figure 1. Tumor-induced bone remodeling, referred pain, and impaired limb use. $\boldsymbol{A}$, Representative radiographs demonstrating significant bone loss in male and female rats with fractures developing within $12 \mathrm{~d}$ after injection. $\boldsymbol{B}, \boldsymbol{C}$, Time-dependent development of tactile hypersensitivity (B) and impaired limb use (C). ${ }^{*} p<0.05,{ }^{* *} p<0.01,{ }^{* * *} p<0.001$ versus presurgery BL. Graphs are mean \pm SEM. $n=8$.

spent in the conditioning chambers after movement (Fig. $2 \mathrm{~A}$, $p>0.05$ vs 0$)$.

To determine whether movement-induced BTP was observed in morphine-treated rats, rats underwent a single-trial conditioning protocol as outlined by the flowchart in Figure $2 \mathrm{~B}$. Continuous administration of morphine across $24 \mathrm{~h}$ failed to block movement-induced CPA (Fig. 2B), indicating that movementinduced pain breaks through morphine previously demonstrated 
to block tumor-induced ongoing pain (Remeniuk et al., 2015). Both placebo and morphine-treated tumor-bearing rats spent significantly decreased amounts of time in the movement-paired chamber compared with sham rats (Fig. $2 B,{ }^{\star} p<0.05$ vs sham-placebo, ${ }^{* * *} p<0.001$ vs shammorphine). The degree of CPA did not differ between the placebo and morphine-treated tumor-bearing rats $(p>0.05$, Bonferroni $t$ test). Sham controls did not demonstrate a decrease in time spent in the movementpaired chamber regardless of placebo or morphine treatment (Fig. $2 B, p>0.05$ vs 0 ).

Blockade of sensory afferent input from tibia prevents, but does not reverse, movement-induced BTP.

To determine whether saphenous lidocaine induced peripheral nerve block prevented movement-induced BTP, rats underwent a single-trial conditioning protocol as outlined by the flowchart in Figure $3 A$. Pretreatment with saline did not alter movement-induced CPA in tumor-bearing rats (Fig. $3 A,{ }^{\star} p<0.05$ vs sham saline). In contrast, pretreatment with lidocaine 10 min before movement of the cancerbearing hindlimb produced CPP (Fig. $3 A$, ${ }^{* * *} p<0.001$ vs sham lidocaine), likely indicating alleviation of tumor-induced ongoing pain. Sham control rats did not alter time spent in the movement-paired chamber after saline or lidocaine treatment 10 min before hindlimb movement (Fig. 3A, $p>0.05$ vs 0$)$.

To determine whether saphenous lidocaine induced peripheral nerve block after movement blocked BTP, rats underwent a single-trial conditioning protocol as outlined by the flowchart in Figure $3 B$. Blockade of afferent input from the tibia by lidocaine administration to the saphenous nerve $10 \mathrm{~min}$ after hindlimb movement did not reverse movement-induced CPA (Fig. 3B). Tumorbearing rats show equivalent decreases in time spent in the movement-paired chamber after saline or lidocaine administration 10 min after movement (Fig. $3 B,{ }^{*} p<0.01$ vs sham-saline, ${ }^{\star} p<$ 0.05 vs sham-lidocaine). Sham rats did not demonstrate CPA to the movement-paired chamber regardless of saline or lidocaine treatment 10 min after movement (Fig. $3 B, p>0.05$ vs 0 ).

Spinal capsaicin eliminates TRPV1, SP, and CGRP immunofluorescence and IB4-SAP diminishes IB4 immunofluorescence in the spinal dorsal horn Immunofluorescent imaging was focused on lamina corresponding to lamina I-IV/V as indicated in the map in Figure $4 A$. Highresolution images of lamina I-II demonstrate that DAPI immunofluorescence (blue) does not overlap with IB4 (Fig. 4B-D, green) or TRPV1 (Fig. 4B, red), SP (Fig. 4C, red), or CGRP (Figure $4 D$ ). A representative image demonstrating the ROIs used to calculate intensity ratios for immunofluorescence in lamina I (TRPV1, SP, or CGRP; L1) and lamina 2 (IB4; L2) and the corresponding control regions (L1con, L2con) in the deep dorsal horn are shown in Figure 4E. Representative images demonstrat- ing coimmunofluorescent stains for TRPV1 (red)/IB4 (green) (Fig. 4F), SP/IB4 (Fig. 4G), and CGRP/IB4 (Fig. 4H) in the spinal dorsal horn from control-treated rats demonstrate similar immunofluorescent patterns to previous reports (Yaksh et al., 1979; Vulchanova et al., 2001; Cavanaugh et al., 2009). Immunofluorescence for TRPV1, SP, and CGRP appear in the lamina I region and IB4 immunofluorescence is observed in lamina II region of the spinal cord dorsal horn. Representative images of coimmunofluorescent stains for TRPV1 and IB4 in the spinal dorsal horn of an IB4-SAP treated rat (Fig. 4I) demonstrate that IB4-SAP diminished IB4 immunofluorescence, as reported previously (Vulchanova et al., 2001). Representative images from spinal dorsal horns of capsaicin-treated rats demonstrate elimination of terminals expressing TRPV1 (Fig. 4J), SP (Fig. 4K), and CGRP (Fig. 4L) without significant alteration of IB4 immunofluorescence.

Intensity ratios of immunofluorescence confirmed that IB4-SAP diminished IB4 immunofluorescence significantly compared with control samples (Fig. $4 M,{ }^{\star} p<0.05$ vs control). IB4-SAP did not alter immunofluorescence for TRPV1 immunofluorescence (Fig. $4 M)$. TRPV1 intensity ratios confirm that capsaicin eliminated TRPV1 immunofluorescence, with values dropping to $96.21 \pm$ $12.22 \%$ of the internal control ROI (Fig. $4 N,{ }^{* *} p<0.01$ vs control). Capsaicin did not alter the immunofluorescence of IB4 (Fig. $4 N, p>0.05$ vs control). SP intensity ratios demonstrate that capsaicin eliminates SP values (Fig. $4 O,{ }^{* *} p<0.01$ vs con- 
A
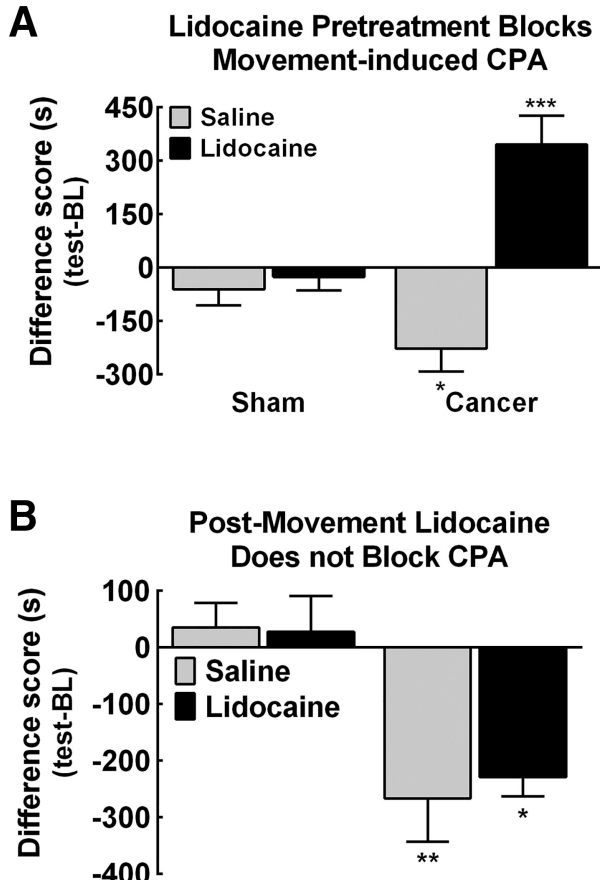

Sham

Cancer

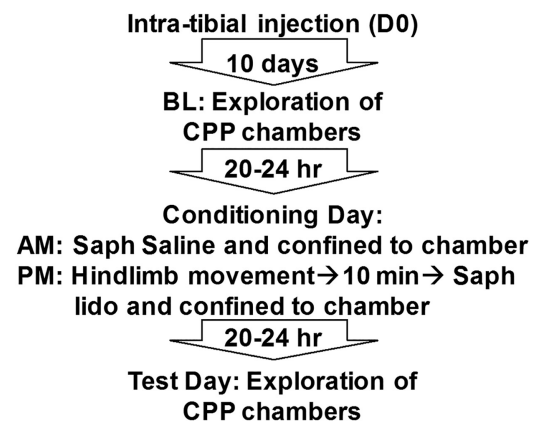

Figure 3. Movement-induced BTP is prevented, but not reversed, by saphenous lidocaine. $A$, Group comparison of difference scores demonstrating that movement of the tumor-bearing hindlimb 10 min after saphenous saline induced CPA in tumor-bearing rats. ${ }^{*} p<0.05$ versus sham saline. Pretreatment with lidocaine $10 \mathrm{~min}$ before movement resulted in a significant increase in time spent in the movement-paired chamber. ${ }^{* *} p<0.001$ versus sham saline. Neither saline nor lidocaine treatment 10 min before movement altered time spent in the movement-paired chamber in sham rats. $n=7 /$ group. $\boldsymbol{B}$, Group comparison of difference scores showing that cancer-treated rats demonstrated equivalent decreases in time spent in the movement-paired chamber when treated with saline or lidocaine 10 min after movement. ${ }^{* *} p<0.01$ versus sham saline, ${ }^{*} p<0.05$ versus sham lidocaine. Neither saline nor lidocaine treatment $10 \mathrm{~min}$ after movement altered time spent in the movement-paired chamber in sham rats. Graphs are mean \pm SEM. $n=5$ /group sham rats; $n=7 /$ group tumor-bearing rats. vehicle), and not significantly different from pretumor baselines (Fig. $5 A, p>$ 0.05 vs $\mathrm{BL})$.

The effects of capsaicin-induced elimination of TRPV $1^{+}$fibers or IB4SAP ablation of IB4-binding fibers on movement-evoked BTP was determined using a single-trial conditioning protocol after spinal injection of capsaicin, IB4SAP or the appropriate vehicle as outlined by the flow-chart (Fig. 5B). Movement failed to induce CPA in sham-treated rats regardless of spinal treatment with capsaicin or IB4-SAP (Fig. $5 B, p>0.05$ vs 0 ). Cancer-treated rats that received spinal administration of the appropriate intrathecal vehicle demonstrated movementinduced CPA as demonstrated by a significantly lower difference score compared with the sham-treated rats (Fig. $5 B$, ${ }^{\star} p<0.05$ vs sham). Tumor-bearing rats that received spinal capsaicin demonstrated movement-induced CPA represented by a significantly lower difference score compared with sham-treated rats (Fig. $5 B,{ }^{* *} p<0.01$ vs sham). Notably, the difference scores of the spinal vehicle and spinal capsaicin-treated tumor-bearing rats did not differ $(p>0.05$, Bonferroni $t$ test). Cancer-bearing rats that received spinal IB4-SAP failed to show movementinduced CPA, as demonstrated by difference scores that did not differ from sham control rats (Fig. $5 B, p>0.05$ ). trol), with mean intensity ratio dropping to $124.71 \pm 11.19 \%$ of the control ROI, a value not different from $100 \%$ ( $p>0.05$ vs null hypothesis). Capsaicin did not alter IB4 immunofluorescence compared with control-treated samples (Fig. 4O, $p>0.05$ vs control). CGRP intensity ratios demonstrate that capsaicin diminishes CGRP values significantly compared with controltreated samples (Fig. $4 P,{ }^{\star} p<0.05$ vs control), although the values remain significantly elevated compared with $100 \%$ control ROI ( $p<0.05$ vs null hypothesis). IB4-SAP did not alter immunofluorescence of CGRP compared with control-treated samples (Fig. 4P).

Functional blockade of IB4-binding, not TRPV1-expressing, fibers blocks BTP

The effects of capsaicin-induced elimination of TRPV ${ }^{+}$fibers or IB4-SAP ablation of IB4-binding fibers on tumor-induced tactile hypersensitivity were measured as outlined by the flowchart in Figure 5A. Cancer-induced tactile hypersensitivity was blocked by spinal administration of IB4-SAP, but not spinal capsaicin (Fig. 5A). Tumor-bearing rats that had received spinal administration of vehicle or SAP developed tactile hypersensitivity by $\mathrm{d}$ 12 after cancer implantation (Fig. $5 A,{ }^{* *} p<0.001$ vs $\mathrm{BL}$ ). Tumor-bearing rats treated with spinal capsaicin demonstrated similar tactile hypersensitivity compared with the vehicle control (Fig. $5 A,{ }^{* * *} p<0.001$ ). In contrast, rats treated with spinal IB4SAP demonstrated tactile withdrawal thresholds that were significantly higher than the vehicle treated rats (Fig. $5 A,{ }^{\#} p<0.05$ vs

\section{Ablation of IB4 or TRPV1-expressing fibers did not alter tumor-induced bone remodeling.}

Radiograph images show representative bone remodeling illustrating the rating scale that was used to determine tumor-induced bone remodeling d 13 after surgery (Fig. 6A). Rats treated with spinal capsaicin or with IB4-SAP did not demonstrate altered bone remodeling compared with their respective vehicle controls (Fig. 6B).

\section{Discussion}

We have developed and characterized a novel measure of movementevoked BTP in the setting of morphine-controlled ongoing pain in a rat model of cancer-induced bone pain. This measure uses the motivational aspects of pain averseness to capture a movement-triggered transient increase in the apparent intensity of cancer-induced bone pain. When this event is paired with a distinctive context, rats show avoidance of the chamber in a subsequent trial producing CPA. This approach is consistent with clinical observations in which patients with bone metastases report transient increases in pain intensity that can be triggered by voluntary (e.g., switching positions) or involuntary (e.g., cough) movements. Notably, this is relatively common in patients with skeletal metastases and can diminish the daily activity of these patients due to their desire to avoid triggering BTP, greatly diminishing quality of life (Mercadante, 2015).

Reverse translation of BT pain

Hindlimb movement before placement into the pairing chamber produced CPA selectively in tumor-bearing rats, indicating that 
A
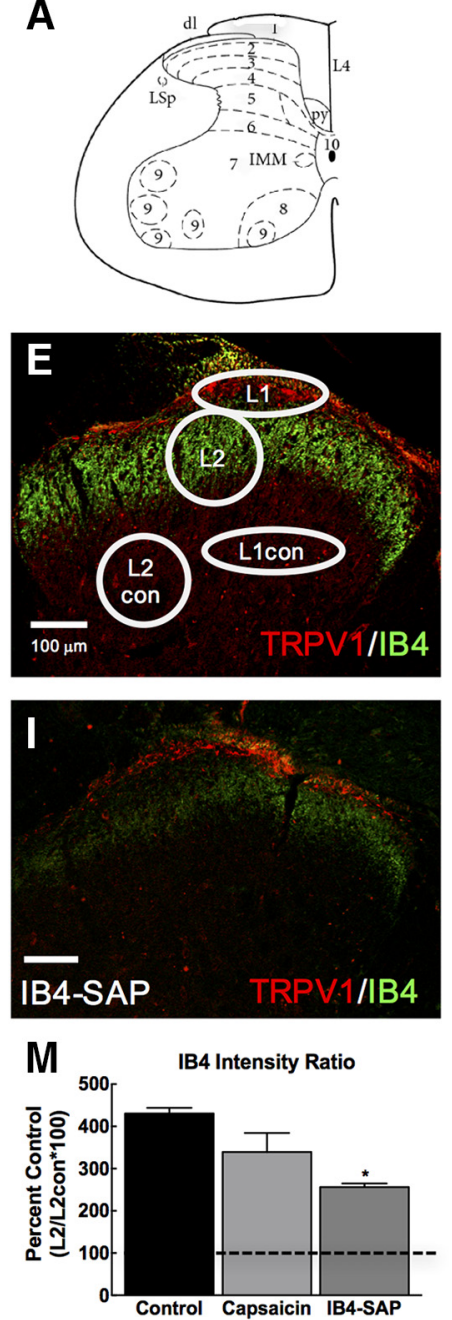
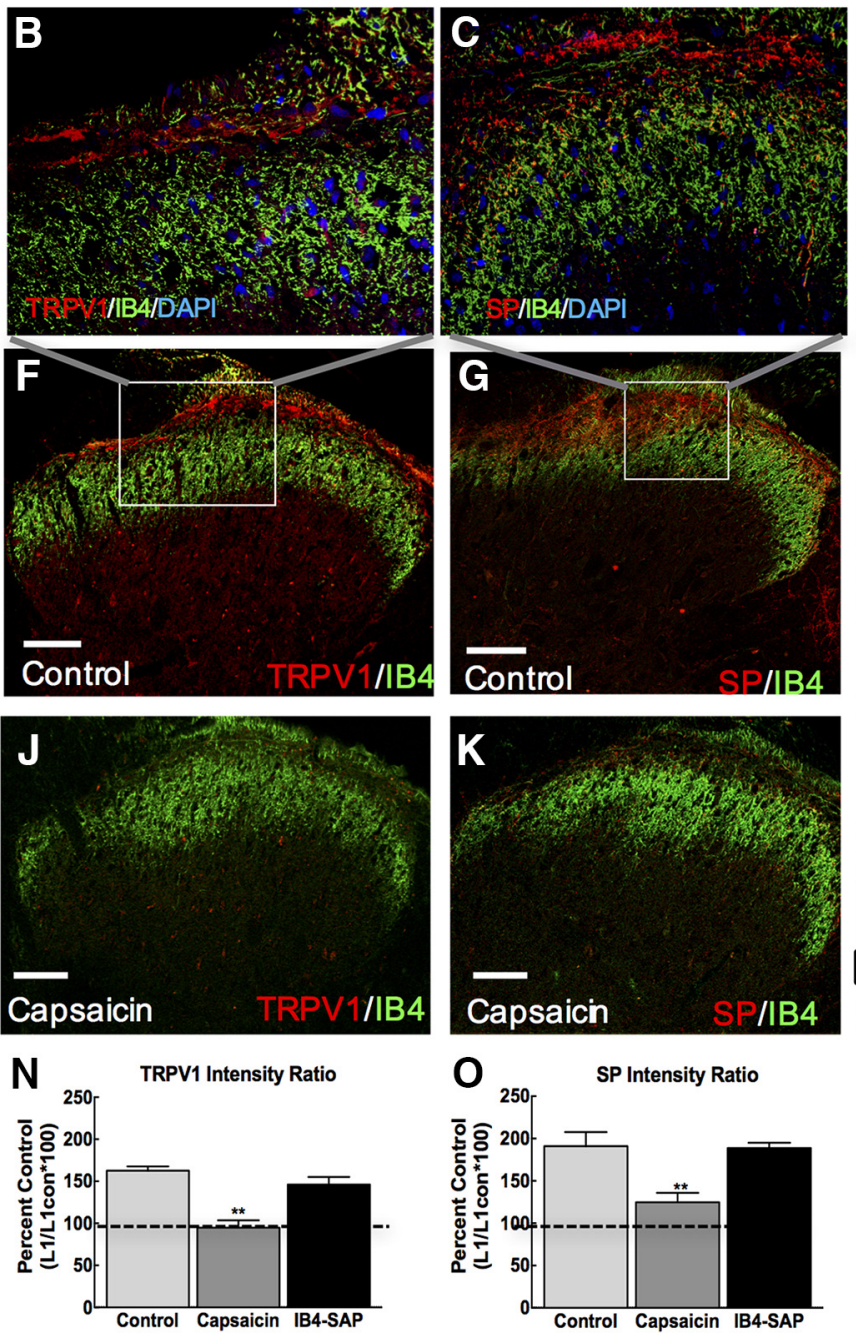
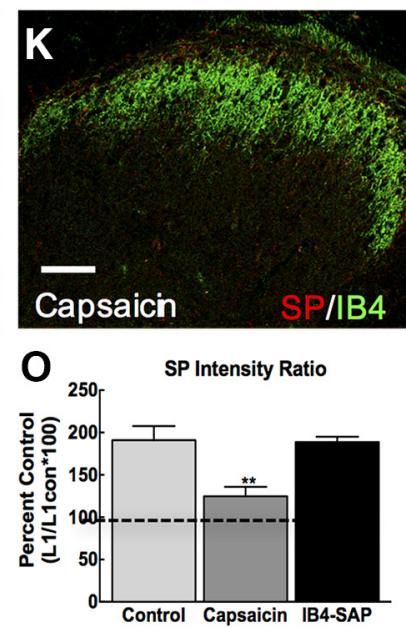
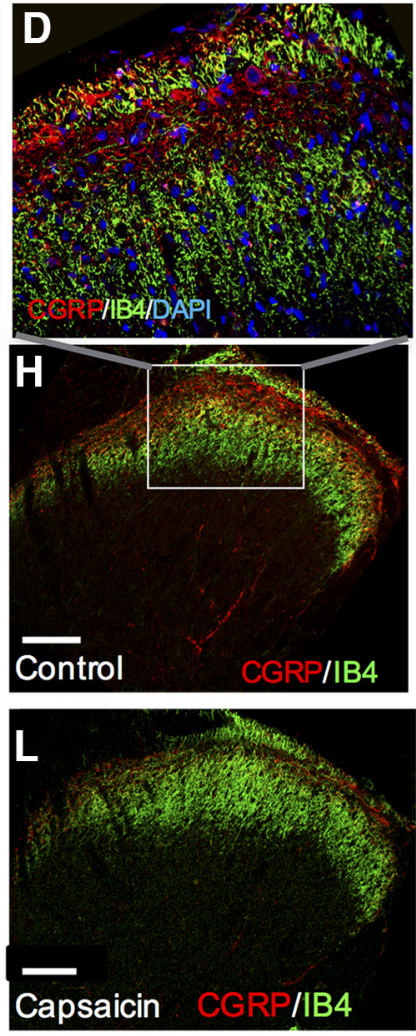

$\mathbf{P}$

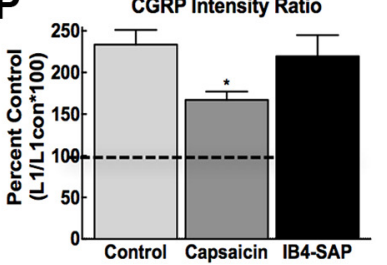

Figure 4. Capsaicin eliminates TRPV1, SP, and CGRP immunofluorescent staining and IB4-SAP diminishes IB4 immunofluorescence in the spinal dorsal horn. $A$, Spinal map from The Rat Brain in Stereotaxic Coordinates, 4th edition (Paxinos and Watson, 1998). B, Representative 40× image inset from $\boldsymbol{F}$ of DAPI (blue), IB4 (green) conjugate fluorescence, and TRPV1 (red) immunofluorescence indicating a lack of overlap between IB4, TRPV1, and DAPI signal in a control animal. Images were collected using a single optical section. C, Representative 40X image inset from $\mathbf{G}$ of DAPI (blue), IB4 (green) conjugate fluorescence, and SP (red) immunofluorescence indicating a lack of overlap between IB4 SP and DAPI signal in a control animal. $\boldsymbol{D}$, Representative $40 \times$ image inset from $\boldsymbol{H}$ of DAPI (blue), IB4 (green) conjugate fluorescence, and GRP (red) immunofluorescence indicating a lack of overlap between IB4, CGRP, and DAPI signal in a control animal. $\boldsymbol{E}$, Representative image demonstrating ROIs used for calculation of intensity ratios. For TRPV1, SP, and CGRP, intensity values from an oval ROl targeting a section of lamina 1 (L1) was divided by intensity values from an oval $\mathrm{RO}$ in the deeper lamina L1 control (L1con). For IB4 immunofluorescence, a circle targeting a section of lamina 2 (L2) was divided by L2 control (L2con). This provides a ratio of the target area of interest divided by an internal control. $\boldsymbol{F}$, Representative 20X image demonstrating TRPV1 (red) and IB4 (green) immunofluorescence in a section from a control rat. G, Representative 20X image demonstrating immunofluorescence for SP (red) and IB4 (green) in a spinal cord section from a control-treated rat. $\boldsymbol{H}$, Representative $20 \times$ image demonstrating immunofluorescence for (GRP (red) and IB4 (green) in a spinal cord section from a control-treated rat. $I$, Representative image demonstrating immunofluorescence of TRPV1 (red) and reduction of IB4 (green) immunofluorescence. $\boldsymbol{J}$, Representative image demonstrating lack of TRPV1 (red) immunofluorescence after treatment with capsaicin with IB4 (green) immunofluorescence remaining. $\boldsymbol{K}$, Representative image demonstrating lack of SP (red) immunofluorescence after treatment with capsaicin with IB4 (green) immunofluorescence remaining. $L$, Representative image demonstrating absence of CGRP (red) immunofluorescence in capsaicin-treated rats with IB4 (green) immun ofluorescence remaining. $M$, Intensity ratios demonstrating that there is a significant reduction in IB4-binding fibers within the spinal cord of IB4-SAP-treated rats compared with controls. No significant difference was observed in capsaicin-treated rats compared with controls. All dashed lines represent ratio value (100) at which there is equivalent mean gray values for $\mathrm{L} 1 / \mathrm{L} 1$ con or $\mathrm{L} 2 / \mathrm{L} 2$ con. Graphs are mean $\pm \mathrm{SEM} .{ }^{*} p<0.05$ versus control, ${ }^{* *} p<0.01$ versus control. $\boldsymbol{N}$, Intensity ratios demonstrate that there is a significant reduction in TRPV1 immunofluorescence in capsaicin-treated rats compared with controls. No difference in TRPV1 intensity ratios were observed in IB4-SAP treated rats. $\mathbf{0}$, Intensity ratios demonstrate a significant reduction in SP immunofluorescence compared with control; no difference in SP immunofluorescence is observed in IB4-SAP treated rats compared with controls. $P$, Intensity ratios demonstrating a significant reduction in CGRP immunofluorescence in capsaicin-treated rats compared with controls, with no significant change in CGRP immunofluorescence in the IB4-SAP-treated rats. Scale bar, $100 \mu \mathrm{m}$.

the movement-triggered increase in pain is aversive and provides learning that motivates animals to avoid that chamber. Movementinduced CPA is observed in rats undergoing morphine treatment previously demonstrated to control tumor-induced ongoing pain (Remeniuk et al., 2015). The defining feature of BTP is that it occurs in patients on opioid medication that is controlling the persistent background pain (Portenoy and Hagen, 1989, 1990; Mercadante, 2015). Moderate to severe cancer pain is often treated with extended-release opioids that engage the MOR (Paice and Ferrell, 2011; Mercadante, 2015). Therefore, our observations show reverse translation from the clinical setting to the rat model of cancer-induced bone pain. Opioids predominately act to modulate affective dimensions of pain by actions at opioid receptors within the brain (Fields, 2004; Navratilova et al., 2015). In addition, MOR agonists may act at receptors localized on primary afferent nociceptors and in the spinal cord (Fields, 2004; 
A

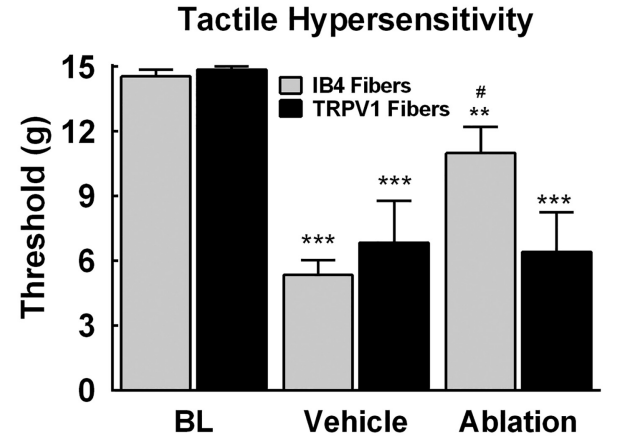

B

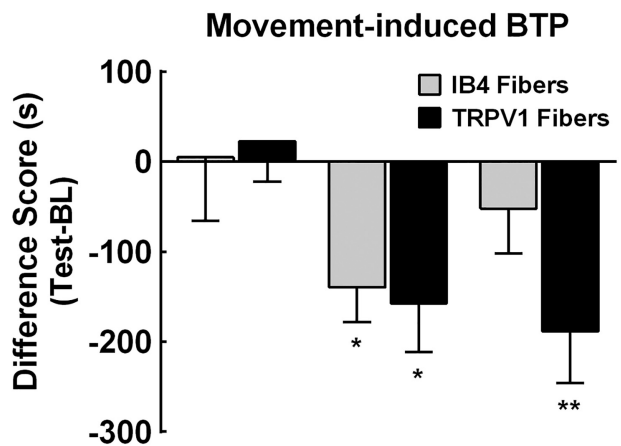

Sham Vehicle Ablation
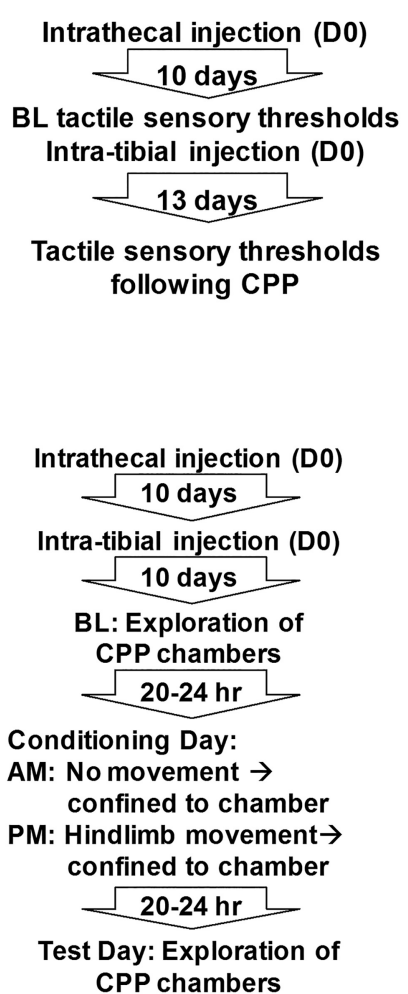

Figure 5. Tactile hypersensitivity and movement-induced BTP is dependent on IB4 positive fibers. A, Spinal administration of IB4-SAP attenuated tumor-induced tactile hypersensitivity. Vehicle-treated tumor-bearing rats demonstrated tactile hypersensitivity with paw withdrawal thresholds significantly lower than precancer implantation baselines. ${ }^{* * *} p<0.01$ versus BL. Ablation of IB4-binding fibers by spinal administration of IB4-SAP attenuated tumor-induced tactile hypersensitivity, with paw withdrawal thresholds significantly higher compared with SAP control rats. \#p $<0.05$ versus SAP. Spinal ablation of TRPV1-expressing terminals in the spinal dorsal horn by spinal administration of capsaicin failed to eliminate tumor-induced tactile hypersensitivity, with paw withdrawal thresholds significantly lower than precancer baselines. ${ }^{* * *} p<0.01$ versus BL. B, Group comparison of difference scores demonstrates that movement induced CPA in vehicle-treated rats compared with sham controls. ${ }^{*} p<0.05$ versus sham. Ablation of IB4-binding fibers blocked movement-induced CPA. In contrast, ablation of TRPV1-expressing terminals in the spinal dorsal horn failed to block movement-induced CPA. ${ }^{* *} p<0.01$ versus shams. Graphs are mean \pm SEM. Sham, $n=$ 10 capsaicin, $n=10$ IB4-SAP; cancer, $n=9$ SAP, $n=8$ capsaicin vehicle, $n=11$ IB4-SAP, and $n=10$ capsaicin.

Ossipov et al., 1999). The clinical observations and our data indicate that movement likely engages additional nociceptive drive that is insensitive to MOR agonists at doses that control ongoing pain.

\section{Potential role of peripheral and central sensitization}

Nociceptors are likely to be sensitized as a consequence of tumoror immune-derived factors within the intramedullary space (Mantyh, 2013; Falk and Dickenson, 2014; Mantyh, 2014). In addition, the ongoing pain from the tumor-bearing bone can produce spinal sensitization (Urch et al., 2003; Yanagisawa et al., 2010; Falk and Dickenson, 2014; Mantyh, 2014). We propose that both peripheral and central sensitization amplifies nociceptive input from movement-evoked stimulation of the tumor-bearing limb, resulting in a transient increase in pain intensity that occurs in the setting of opioids controlling the persistent background ongoing pain. Preclinical studies demonstrated that spinal cord neurons show enhanced responses to evoked stimuli and wide dynamic range neurons display an increase in receptive field, hallmarks of spinal sensitization (Urch et al., 2003; Falk and Dickenson, 2014). These changes are observed in the setting of morphine and have been proposed to underlie opioid resistant allodynia and BTP (Urch et al., 2005). Such signaling likely increases the intensity of pain experienced, thereby also increasing the affective/motivational component of the tumor-induced pain (Fields, 1999). The transient increase in pain and related unpleasantness surpasses the ability of the onboard dose of morphine to control the pain, resulting in the requirement for additional rapid onset opioids as seen in the clinical setting: BTP.

\section{Mechanistic difference between}

initiation and maintenance of BTP

Sensory input from the tumor-bearing bone is required for initiation of BTP. Saphenous nerve block administered $10 \mathrm{~min}$ before movement of the tumor-bearing hindlimb prevented movement-induced BTP and induced CPP. This observation indicates that the peripheral nerve block not only prevented the movement-triggered BT pain, but also blocked tumor-induced ongoing pain. Therefore, the rats experience pain relief in the lidocaine/movement paired chamber. Therefore, pain relief is the motivating factor that results in the increased time spent in the chamber paired with nerve block and movement. This replicated our previous findings that peripheral nerve block produces relief from tumor-induced ongoing bone pain (Remeniuk et al., 2015). In contrast, blockade of sensory input after hindlimb movement failed to block CPA. These observations suggest that, once established, blocking sensory input from the tumorbearing bone is no longer sufficient to reverse movement-induced BTP. We speculate that one possible explanation is the engagement of reverberating circuits that maintain activity within the spinal cord or between the spinal cord and central nuclei independently from peripheral input. Such altered processing could account for prolonged withdrawal responses to noxious pinprick as reported after chronic constriction injury of the sciatic nerve (Bennett and Xie, 1988). A reverberating circuit between spinal cord dorsal horn and the dorsal reticular nucleus within the caudal medulla has been proposed to promote enhanced response capacity of spinal neurons to noxious stimulation and has been implicated in acute pain responses to noxious heat and formalin (Lima and Almeida, 2002). Such reverberating circuitry may be critical in maintaining BTP and deserves further study.

Separate roles of TRPV1 ${ }^{+}$and IB4-binding fibers in BT pain The observation that saphenous nerve block prevents movementinduced CPA suggests that the initiation of BTP is dependent on sensory input from the tumor-bearing bone. Our data indicate that IB4-binding fibers mediate movement-induced breakthrough cancer pain and referred tactile hypersensitivity, whereas TRPV $1^{+}$fibers do not. These observations are consistent with studies demonstrating that functional blockade of TRPV1 ${ }^{+} \mathrm{fi}^{-}$ bers fails to alter responses to noxious mechanical stimulation 
A

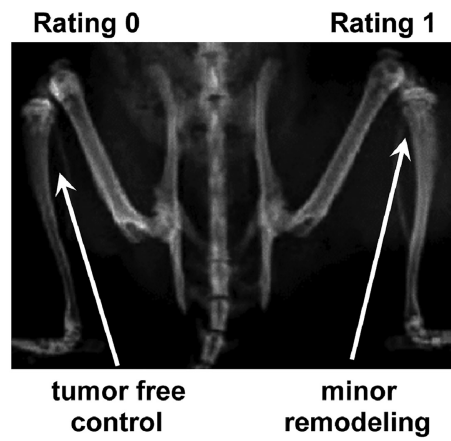

Rating 2

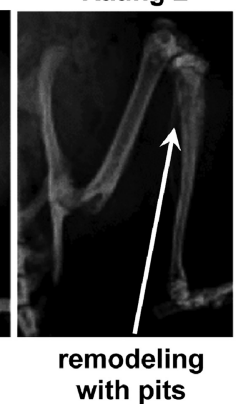

Rating 3

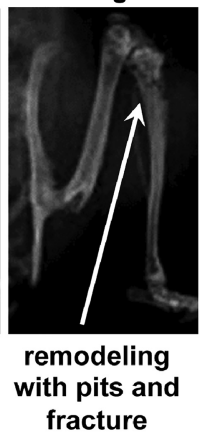

B

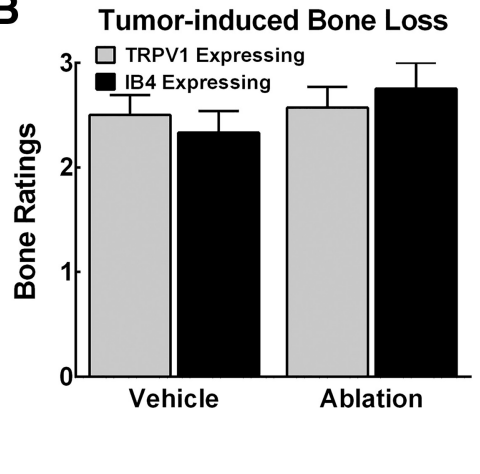

Figure 6. Tumor-induced bone loss is observed in all tumor-bearing rats regardless of treatment. $\boldsymbol{A}$, Representative images of bone radiographs demonstrating ratings using a 4-point scale described in the text. $\boldsymbol{B}$, All treatment groups demonstrated equivalent levels of bone loss regardless of spinal treatment. Graphs are mean \pm SEM.

and tactile hypersensitivity (Ossipov et al., 1999; Cavanaugh et al., 2009; Scherrer et al., 2009; Okun et al., 2011). Our findings are also consistent with studies demonstrating that ablation of IB4binding fibers blocks tactile hypersensitivity in preclinical models of inflammation, neuropathic pain, and cancer-induced orofacial pain (Stucky and Lewin, 1999; Vulchanova et al., 2001; Joseph et al., 2008; Cavanaugh et al., 2009; Ye et al., 2012). Given these data, we propose that movement recruits TRPV1 ${ }^{-}$nociceptive fibers, resulting in an increased pain signal that initiates an episode of BTP that is not adequately prevented by peripheral or central actions of opioids used to control background persistent ongoing pain.

After capsaicin treatment, we saw reduced CGRP and complete elimination of SP immunofluorescence and TRPV1 ${ }^{+}$fiber terminals in the spinal dorsal horn, observations consistent with previous studies (Yaksh et al., 1979; Cavanaugh et al., 2009). IB4Saporin eliminated IB4-staining indicating elimination of input from IB4-binding sensory afferent fibers. The absence of sensory input by these nerve terminals within the L3 segment of the lumbar spinal cord likely eliminates much of the sensory input from the saphenous nerve, previously demonstrated to be the primary innervation for the rat tibia (Ivanusic, 2009; Kaan et al., 2010). Previous studies have suggested a lack of IB4-binding fibers in mouse models of cancer-induced bone pain (Jimenez-Andrade et al., 2010; Castañeda-Corral et al., 2011). In contrast, retrograde labeling techniques indicate that IB4-binding fibers do innervate the intramedullary space and the periosteum of the rat tibia (Ivanusic, 2009; Kaan et al., 2010). It is unclear whether the discrepancies between these reports are due to methodological differences or species differences. Regardless of whether IB4binding fibers innervate the bone, it is likely that sensory input from tissue surrounding the bone may also contribute to the initiation of movement-evoked BTP.

Spinal capsaicin was demonstrated to reduce SP content within sensory fibers within $7 \mathrm{~d}$ (Yaksh et al., 1979). Such observations indicate that the capsaicin-induced blockade of sensory input by TRPV $1{ }^{+}$fibers in our studies occurred before tumor cell injection and subsequent growth of tumor cells within the tibia, thereby eliminating ongoing input from TRPV $1^{+}$nociceptive fibers throughout the experiment. Our data demonstrate that elimination of signaling by TRPV $1^{+}$fibers is not sufficient to block movement-induced CPA or referred pain as measured by tactile hypersensitivity in the ipsilateral hindpaw. This is consistent with other observations that long-term desensitization of TRPV1-expressing fibers by systemic administration of the ultrapotent capsaicin analog resiniferotoxin blocks thermal and ongoing pain, but fails to block development of tactile hypersensitivity in other chronic pain models (Ossipov et al., 1999; King et al., 2011; Okun et al., 2011). Our observations of hindpaw tactile hypersensitivity, a measure of referred pain, and initiation of BTP by a normally non-noxious stimulus suggest that the tumorbearing rats developed central sensitization in the absence of input from TRPV1-expressing fibers. Previous studies have demonstrated that the development of central sensitization is dependent on input from nociceptive afferents, presumably C-fibers (Gracely et al., 1992; Sang et al., 1996). It is likely that sustained input from non-TRPV1 nociceptive fibers is sufficient to develop central sensitization that mediates hypersensitivity to nonnoxious mechanical stimulation.

Further research is warranted to examine subpopulations of sensory fibers in relation to bone pain. As noted above, single-cell RNAseq studies have demonstrated that there are many potential subcategories of sensory fibers, with as many as 11 types of sensory neurons in the mouse DRG (Usoskin et al., 2015). Moreover, some studies have indicated overlap of CGRP in IB4-binding neurons in species and site-specific patterns (Aoki et al., 2005; Hwang et al., 2005; Price and Flores, 2007). Future studies using techniques such as single-cell capture and RNAseq of DRG cells that have been retrogradely labeled from the bone, periosteum, and perhaps the surrounding tissue are necessary to clarify potential subpopulations of IB4-binding neurons that may mediate BT pain. In addition, studies examining corresponding protein expression and the relative functional contribution of observed subpopulations of fibers innervating the bone are necessary.

Our data indicate distinctive mechanisms underlying tumorinduced ongoing and BTP. As with all preclinical studies, future studies are required to show reproducibility of these findings across different strains and species. This highlights the need to determine whether therapeutic strategies currently under development block both ongoing and BTP. Notably, advances in nonopioid therapies for ongoing pain are urgently needed to diminish reliance on opioids regardless of whether they effectively block BTP. Alternatively, novel compounds targeting IB4-binding nociceptors may improve pain management for cancer pain patients and other patient populations suffering from BTP that is inadequately treated by currently available medications.

\section{References}

Aoki Y, Ohtori S, Takahashi K, Ino H, Douya H, Ozawa T, Saito T, Moriya H (2005) Expression and co-expression of VR1, CGRP, and IB4-binding glycoprotein in dorsal root ganglion neurons in rats: differences between the disc afferents and the cutaneous afferents. Spine 30:1496-1500. CrossRef Medline 
Basbaum AI, Bautista DM, Scherrer G, Julius D (2009) Cellular and molecular mechanisms of pain. Cell 139:267-284. CrossRef Medline

Bennett GJ, Xie YK (1988) A peripheral mononeuropathy in rat that produces disorders of pain sensation like those seen in man. Pain 33:87-107. CrossRef Medline

Bennett MI (2010) Cancer pain terminology: time to develop a taxonomy that promotes good clinical practice and allows research to progress. Pain 149:426-427. CrossRef Medline

Breivik H, Cherny N, Collett B, de Conno F, Filbet M, Foubert AJ, Cohen R, Dow L (2009) Cancer-related pain: a pan-European survey of prevalence, treatment, and patient attitudes. Ann Oncol 20:1420-1433. CrossRef Medline

Castañeda-Corral G, Jimenez-Andrade JM, Bloom AP, Taylor RN, Mantyh WG, Kaczmarska MJ, Ghilardi JR, Mantyh PW (2011) The majority of myelinated and unmyelinated sensory nerve fibers that innervate bone express the tropomyosin receptor kinase A. Neuroscience 178:196-207. CrossRef Medline

Cavanaugh DJ, Lee H, Lo L, Shields SD, Zylka MJ, Basbaum AI, Anderson DJ (2009) Distinct subsets of unmyelinated primary sensory fibers mediate behavioral responses to noxious thermal and mechanical stimuli. Proc Natl Acad Sci U S A 106:9075-9080. CrossRef Medline

Chaplan SR, Bach FW, Pogrel JW, Chung JM, Yaksh TL (1994) Quantitative assessment of tactile allodynia in the rat paw. J Neurosci Methods 53:5563. CrossRef Medline

Falk S, Dickenson AH (2014) Pain and nociception: mechanisms of cancerinduced bone pain. J Clin Oncol 32:1647-1654. CrossRef Medline

Fields H (2004) State-dependent opioid control of pain. Nat Rev Neurosci 5:565-575. Medline

Fields HL (1999) Pain: an unpleasant topic. Pain Suppl 6:S61-S69. Medline Gracely RH, Lynch SA, Bennett GJ (1992) Painful neuropathy: altered central processing maintained dynamically by peripheral input. Pain 51:175194. CrossRef Medline

Haugen DF1, Hjermstad MJ, Hagen N, Caraceni A, Kaasa S; European Palliative Care Research Collaborative (EPCRC) (2010) Assessment and classification of cancer breakthrough pain: a systematic literature review. Pain 149:476-482. CrossRef Medline

Helsby MA, Leader PM, Fenn JR, Gulsen T, Bryant C, Doughton G, Sharpe B, Whitley P, Caunt CJ, James K, Pope AD, Kelly DH, Chalmers AD (2014) CiteAb: a searchable antibody database that ranks antibodies by the number of times they have been cited. BMC Cell Biol 15:6. CrossRef Medline

Hwang SJ, Oh JM, Valtschanoff JG (2005) The majority of bladder sensory afferents to the rat lumbosacral spinal cord are both IB4- and CGRPpositive. Brain Res 1062:86-91. CrossRef Medline

Isensee J, Wenzel C, Buschow R, Weissmann R, Kuss AW, Hucho T (2014) Subgroup-elimination transcriptomics identifies signaling proteins that define subclasses of TRPV1-positive neurons and a novel paracrine circuit. PLoS One 9:e115731. CrossRef Medline

Ivanusic JJ (2009) Size, neurochemistry, and segmental distribution of sensory neurons innervating the rat tibia. J Comp Neurol 517:276-283. CrossRef Medline

Jimenez-Andrade JM, Mantyh WG, Bloom AP, Xu H, Ferng AS, Dussor G, Vanderah TW, Mantyh PW (2010) A phenotypically restricted set of primary afferent nerve fibers innervate the bone versus skin: therapeutic opportunity for treating skeletal pain. Bone 46:306-313. CrossRef Medline

Joseph EK, Chen X, Bogen O, Levine JD (2008) Oxaliplatin acts on IB4positive nociceptors to induce an oxidative stress-dependent acute painful peripheral neuropathy. J Pain 9:463-472. CrossRef Medline

Kaan TK, Yip PK, Patel S, Davies M, Marchand F, Cockayne DA, Nunn PA, Dickenson AH, Ford AP, Zhong Y, Malcangio M, McMahon SB (2010) Systemic blockade of $\mathrm{P} 2 \mathrm{X} 3$ and $\mathrm{P} 2 \mathrm{X} 2 / 3$ receptors attenuates bone cancer pain behaviour in rats. Brain 133:2549-2564. CrossRef Medline

King T, Vardanyan A, Majuta L, Melemedjian O, Nagle R, Cress AE, Vanderah TW, Lai J, Porreca F (2007) Morphine treatment accelerates sarcoma-induced bone pain, bone loss, and spontaneous fracture in a murine model of bone cancer. Pain 132:154-168. CrossRef Medline

King T, Qu C, Okun A, Mercado R, Ren J, Brion T, Lai J, Porreca F (2011) Contribution of afferent pathways to nerve injury-induced spontaneous pain and evoked hypersensitivity. Pain 152:1997-2005. CrossRef Medline

Lima D, Almeida A (2002) The medullary dorsal reticular nucleus as a pronociceptive centre of the pain control system. Prog Neurobiol 66:81-108. CrossRef Medline
Luger NM, Honore P, Sabino MA, Schwei MJ, Rogers SD, Mach DB, Clohisy DR, Mantyh PW (2001) Osteoprotegerin diminishes advanced bone cancer pain. Cancer Res 61:4038-4047. Medline

Mantyh P (2013) Bone cancer pain: causes, consequences, and therapeutic opportunities. Pain 154:S54-S62. CrossRef Medline

Mantyh PW (2014) Bone cancer pain: from mechanism to therapy. Current opinion in supportive and palliative care 8:83-90. CrossRef Medline

Mercadante S (2011) Managing breakthrough pain. Curr Pain Headache Rep 15:244-249. CrossRef Medline

Mercadante S (2015) Breakthrough pain in cancer patients: prevalence, mechanisms and treatment options. Curr Opin Anaesthesiol 28:559-564. CrossRef Medline

Molliver DC, Wright DE, Leitner ML, Parsadanian AS, Doster K, Wen D, Yan Q, Snider WD (1997) IB4-binding DRG neurons switch from NGF to GDNF dependence in early postnatal life. Neuron 19:849-861. CrossRef Medline

Navratilova E, Atcherley CW, Porreca F (2015) Brain circuits encoding reward from pain relief. Trends Neurosci 38:741-750. CrossRef Medline

Okun A, DeFelice M, Eyde N, Ren J, Mercado R, King T, Porreca F (2011) Transient inflammation-induced ongoing pain is driven by TRPV1 sensitive afferents. Mol Pain 7:4. CrossRef Medline

Ossipov MH, Bian D, Malan TP Jr, Lai J, Porreca F (1999) Lack of involvement of capsaicin-sensitive primary afferents in nerve-ligation injury induced tactile allodynia in rats. Pain 79:127-133. CrossRef Medline

Paice JA, Ferrell B (2011) The management of cancer pain. CA Cancer J Clin 61:157-182. CrossRef Medline

Paxinos G, Watson C (1998) The rat brain in stereotaxic coordinates, Ed 4. San Diego: Academic.

Portenoy RK, Hagen NA (1989) Breakthrough pain: definition and management. Oncology (Williston Park) 3:25-29. Medline

Portenoy RK, Hagen NA (1990) Breakthrough pain: definition, prevalence and characteristics. Pain 41:273-281. CrossRef Medline

Price TJ, Flores CM (2007) Critical evaluation of the colocalization between calcitonin gene-related peptide, substance $\mathrm{P}$, transient receptor potential vanilloid subfamily type 1 immunoreactivities, and isolectin $\mathrm{B} 4$ binding in primary afferent neurons of the rat and mouse. J Pain 8:263-272. Medline

Remeniuk B, Sukhtankar D, Okun A, Navratilova E, Xie JY, King T, Porreca F (2015) Behavioral and neurochemical analysis of ongoing bone cancer pain in rats. Pain 156:1864-1873. CrossRef Medline

Sabino MA, Ghilardi JR, Jongen JL, Keyser CP, Luger NM, Mach DB, Peters CM, Rogers SD, Schwei MJ, de Felipe C, Mantyh PW (2002a) Simultaneous reduction in cancer pain, bone destruction, and tumor growth by selective inhibition of cyclooxygenase-2. Cancer Res 62:7343-7349. Medline

Sabino MC, Ghilardi JR, Feia KJ, Jongen JL, Keyser CP, Luger NM, Mach DB, Peters CM, Rogers SD, Schwei MJ, De Filipe C, Mantyh PW (2002b) The involvement of prostaglandins in tumorigenesis, tumor-induced osteolysis and bone cancer pain. J Musculoskelet Neuronal Interact 2:561-562. Medline

Sang CN, Gracely RH, Max MB, Bennett GJ (1996) Capsaicin-evoked mechanical allodynia and hyperalgesia cross nerve territories: Evidence for a central mechanism. Anesthesiology 85:491-496. CrossRef Medline

Scherrer G, Imamachi N, Cao YQ, Contet C, Mennicken F, O'Donnell D, Kieffer BL, Basbaum AI (2009) Dissociation of the opioid receptor mechanisms that control mechanical and heat pain. Cell 137:1148-1159. CrossRef Medline

Schindelin J, Arganda-Carreras I, Frise E, Kaynig V, Longair M, Pietzsch T, Preibisch S, Rueden C, Saalfeld S, Schmid B, Tinevez JY, White DJ, Hartenstein V, Eliceiri K, Tomancak P, Cardona A (2012) Fiji: an opensource platform for biological-image analysis. Nat Methods 9:676-682. CrossRef Medline

Schwei MJ, Honore P, Rogers SD, Salak-Johnson JL, Finke MP, Ramnaraine ML, Clohisy DR, Mantyh PW (1999) Neurochemical and cellular reorganization of the spinal cord in a murine model of bone cancer pain. J Neurosci 19:10886-10897. Medline

Snider WD, McMahon SB (1998) Tackling pain at the source: new ideas about nociceptors. Neuron 20:629-632. CrossRef Medline

Stucky CL, Lewin GR (1999) Isolectin B(4)-positive and -negative nociceptors are functionally distinct. J Neurosci 19:6497-6505. Medline

Urch CE, Donovan-Rodriguez T, Dickenson AH (2003) Alterations in dor- 
sal horn neurones in a rat model of cancer-induced bone pain. Pain 106:347-356. CrossRef Medline

Urch CE, Donovan-Rodriguez T, Gordon-Williams R, Bee LA, Dickenson AH (2005) Efficacy of chronic morphine in a rat model of cancerinduced bone pain: behavior and in dorsal horn pathophysiology. J Pain 6:837-845. CrossRef Medline

Usoskin D, Furlan A, Islam S, Abdo H, Lönnerberg P, Lou D, Hjerling-Leffler J, Haeggström J, Kharchenko O, Kharchenko PV, Linnarsson S, Ernfors P (2015) Unbiased classification of sensory neuron types by large-scale single-cell RNA sequencing. Nat Neurosci 18:145-153. CrossRef Medline

Vulchanova L, Olson TH, Stone LS, Riedl MS, Elde R, Honda CN (2001)

Cytotoxic targeting of isolectin IB4-binding sensory neurons. Neuroscience 108:143-155. CrossRef Medline

Wang H, Zylka MJ (2009) Mrgprd-expressing polymodal nociceptive neu- rons innervate most known classes of substantia gelatinosa neurons. J Neurosci 29:13202-13209. CrossRef Medline

Yaksh TL, Rudy TA (1976) Chronic catheterization of the spinal subarachnoid space. Physiol Behav 17:1031-1036. CrossRef Medline

Yaksh TL, Farb DH, Leeman SE, Jessell TM (1979) Intrathecal capsaicin depletes substance $\mathrm{P}$ in the rat spinal cord and produces prolonged thermal analgesia. Science 206:481-483. CrossRef Medline

Yanagisawa Y, Furue H, Kawamata T, Uta D, Yamamoto J, Furuse S, Katafuchi T, Imoto K, Iwamoto Y, Yoshimura M (2010) Bone cancer induces a unique central sensitization through synaptic changes in a wide area of the spinal cord. Mol Pain 6:38. CrossRef Medline

Ye Y, Dang D, Viet CT, Dolan JC, Schmidt BL (2012) Analgesia targeting IB4-positive neurons in cancer-induced mechanical hypersensitivity. J Pain 13:524-531. CrossRef Medline 